\title{
Practical Tunneling Magnetoresistive Z-Axis Sensors
}

\author{
James G. Deak \\ MultiDimension Technology Co., Ltd., Zhangjiagang, P.R. China, \\ jim.deak@dowaytech.com
}

\begin{abstract}
:
A practical tunneling magnetoresistive magnetic field sensor for detecting the magnetic field component perpendicular to the plane of the sensor substrate (Z-axis) is described. The sensor consists of MgO-based MTJs (magnetic tunnel junctions) interconnected as a full-bridge and combined with an array of on-chip flux concentrators. The flux concentrator array is designed to steer the flux from the vertical direction into the in-plane direction parallel to the surface of the substrate enabling the MTJ full-bridge to exhibit true push-pull operation, while additionally providing a high packing density of MTJ elements, which enables robust and high precision performance. The resulting single-chip sensors can be tuned for high sensitivity exceeding $3 \mathrm{mV} / \mathrm{V} / \mathrm{G}$ or wide dynamic range exceeding $1000 \mathrm{G}$.
\end{abstract}

Key words: magnetic tunnel junction, magnetic field sensor, Z-axis, tunnelling magnetoresistance, flux concentrator.

\section{Z-Axis Magnetic Field Sensing Overview}

With little doubt, the most ubiquitous magnetic field sensors ever developed are Hall Effect sensors. Implemented on a monolithic silicon chip, they naturally sense the Z-axis magnetic field, and many applications have been developed based on this sensing geometry. These sensors have very wide dynamic range, good linearity, and small size. As a result, they have dominated many applications, requiring small form factors, including compassing and linear field sensing. The relationship between the Hall Effect and the Z-axis is illustrated in Fig. 1.

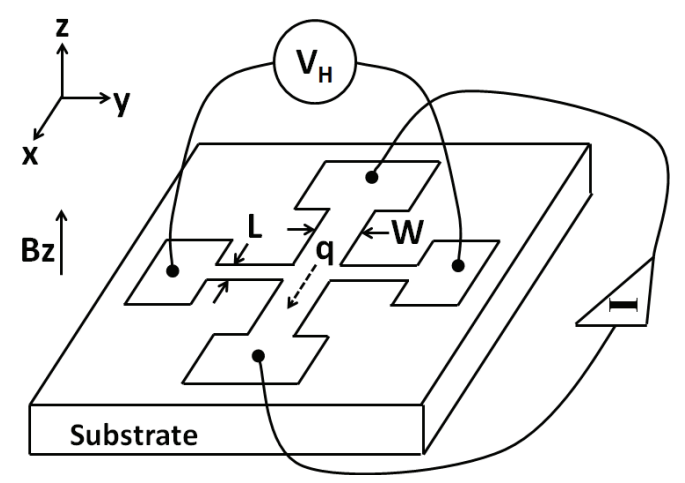

Fig. 1. Schematic drawing of a Hall Effect sensor chip with field applied along the Z-axis and current applied along the $X$-axis. The output voltage is detected across the $Y$-axis.
In this configuration, the charge carriers $q$ (assumed positive) move along the $\mathrm{X}$-axis, and the Z-Axis oriented magnetic field, $B_{z}$, produces a Lorentz force on the carriers along the $Y$-axis, given by

$\vec{F}=q(\vec{E}+\vec{v} \times \vec{B})=0$.

Where, $E=V_{H} / W, v=L / t, I=Q / t, Q=n L W T q$, $q=e, L$ is the conducting channel length, $T$ the channel thickness, $\mathrm{W}$ the channel width, $\mathrm{v}$ the carrier velocity, e is carrier charge, $n$ is carrier density, and $t$ is time. Solving this equation for Hall voltage, yields a Y-directed voltage proportional to $\mathrm{Bz}$,

$V_{H}=-\frac{I B_{z}}{n t e}$.

As can be seen, the Hall Effect sensor utilizes a current in the plane of the substrate to detect a field perpendicular to the substrate. Field detection perpendicular to the substrate may be called Z-axis sensing.

Because Hall Effect sensors utilize Lorentz force and the response is linear with respect to $\mathrm{B}_{\mathrm{z}}$, and Hall Effect sensors have excellent linearity. They do not saturate until $V_{H}$ approaches the bias voltage produced by the bias current, making them an excellent choice for high fields. Unfortunately, the sensor area defined by LW is actually quite small, which makes signal-to-noise poor, and misalignment 
between the voltage taps causes voltage offset. Additionally, the carrier density is quite temperature dependent. As a result, Hall Effect sensors are a poor choice for high precision measurement. Moreover, because high current is needed for high sensitivity, power consumption is high. In summary, Hall Effect sensors suffer from high power consumption, poor noise performance, poor temperature performance, large offset, and low speed as a result of signal processing required for compensating offset.

\section{Magnetoresistance and In-Plane Sensing}

Because of the limitations of Hall Effect sensors, magnetoresistive (MR) sensors have been extensively investigated for higher performance and lower power sensing applications. Magnetoresistive effects include anisotropic magnetoresistance (AMR), giant magnetoresistance (GMR), and tunneling magnetoresistance (TMR).

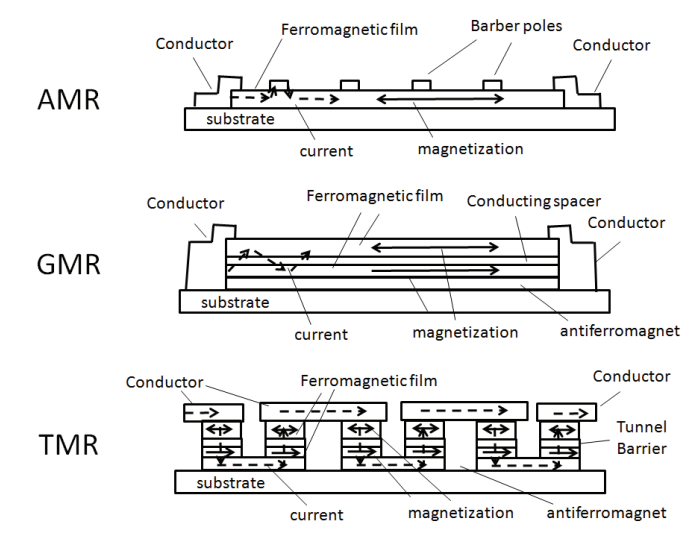

Fig. 2. Layering structure, current path, and magnetization of the 3 different MR effects.

Fig. 2 illustrates the layering sequences of the different MR effects. All of the effects involve at least one ferromagnetic layer that has a magnetization that is free to align parallel to the in-plane component of the applied magnetic field. This ferromagnetic layer is often referred to as the free layer. The GMR and TMR effects include at least one more ferromagnetic layer, that may have its magnetization direction pinned by adding an adjacent antiferromagnetic layer next to it. This layer is often referred to as the pinned layer.

The resistance of an AMR device varies in response the cosine of the square of the angle between the direction of the current and the magnetization, and that of the GMR and TMR effects varies as the square of cosine of the angle between the direction of the bias current and the magnetization of the ferromagnetic layer. The resistance of the GMR and TMR effects varies as the cosine of difference between the angle of the free ferromagnetic layer's and the pinned ferromagnetic layer's magnetization directions. Because thin ferromagnetic films are used, the magnetization remains parallel to the surface of the substrate, and the sensing direction is thus parallel to the surface of the substrate, unless a ferromagnetic material with large perpendicular anisotropy is used.

These MR effects have superior sensitivity and noise performance compared to the Hall Effect, but they have limited dynamic range due to saturation of the ferromagnetic layers. TMR sensors are especially attractive, since they exhibit the highest sensitivity of the magnetoresistive sensors, which is on the order of 1000 times as great as that of a Hall Effect sensor, and TMR sensors can be built with high resistance in order to enable low-power operation. In-plane sensing geometry and dynamic range of MR sensors have protected the Hall Effect sensor's dominance in many general purpose applications, but creative solutions for Z-axis sensing have been devised that are enabling MR sensors to be used in more applications.

\section{Z-axis sensing with MR Sensors}

In order to obtain the benefit of MR sensing in applications that require detection of Z-Axis magnetic fields, several methods have been improvised in commercial products and academic research. Integrated three-axis magnetic sensors such as eCompasses have strongly driven this development. The eCompass market is a highly competitive, and it has pushed MR sensor technology to its limits, by requiring low cost, low power, low noise, and small size. Methods to provide Z-axis sensing in eCompasses include multi-chip modules with a MR sensor die tilted on its side [1], deposition of MR materials on sloped surfaces [2], and perpendicular magnetic anisotropy (PMA) materials [3].

Tilted chips within multi-chip modules have been successful, but it is an expensive approach that produces a relatively large package. In order to develop lower cost single chip sensors, techniques have been developed for depositing and patterning MR materials on sloped surfaces cut into a single semiconductor die. The processes for achieving this goal are not easy, the MR materials do not deposit 
optimally on the sloped surfaces, and the resulting sensors require signal processing to deconvolute the three orthogonal directions which impacts both accuracy and bandwidth. Attempts to produce planar three-axis eCompasses have also led to research on PMA materials. Reported attempts show the PMA method is not yet practical, yielding sensors with large hysteresis, poor sensitivity, or bad stability. [3]

Recently, on-chip concentrators have been shown to achieve good Z-axis magnetic field sensing performance, and this work describes a simple manufacturing process that yields Z-axis TMR sensors with good magnetic sensing performance. [4]

\section{Tilted Chips}

Because MR sensor chips naturally sense inplane magnetic fields, that is, magnetic fields parallel to the surface of the chip, the most straightforward approach to fabricating a Z-Axis magnetic field sensor is to mechanically tilt the Z-Axis MR chip on it side. This can either be done by mounting the MR chips in a SIP style package where the package is tilted as shown in Fig. 3, or by tilting the sensor chips on their side within a package, as in Fig. 4. These approaches are large and relatively expensive to implement a full three-axis sensor.
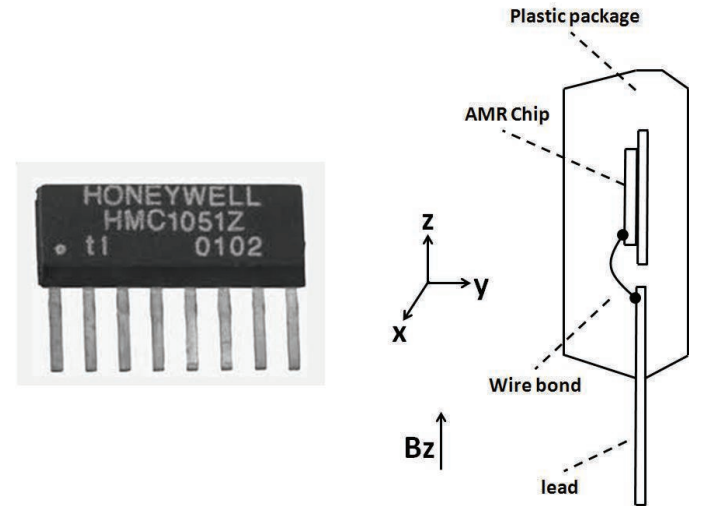

Fig. 3. A Honeywell HMC1051Z AMR Z-axis sensor, which is formed by packaging an in-plane sensing AMR chip in a single in-line package.

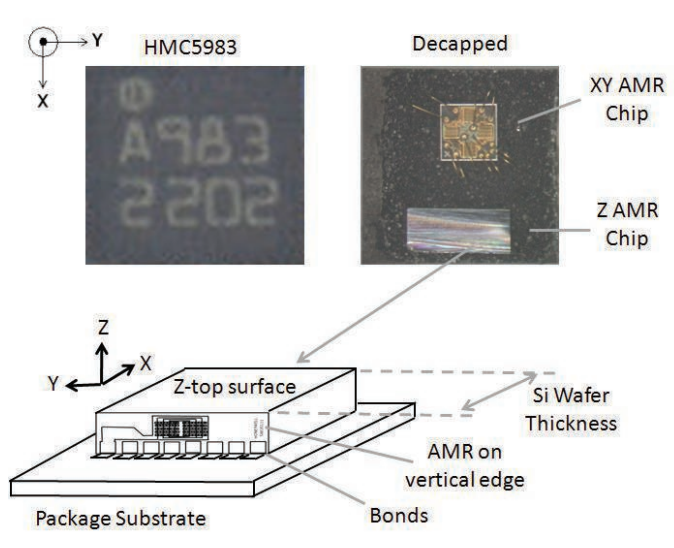

Fig. 4. A Honeywell HMC5983 three-axis AMR sensor, multi-chip package wherein the Z-axis sensor, shown on larger scale at the bottom, is an AMR die that is tilted on edge, and the bond pads of the tilted AMR die meet at a 90 degree angle with the package substrate.

\section{Sloped Surfaces}
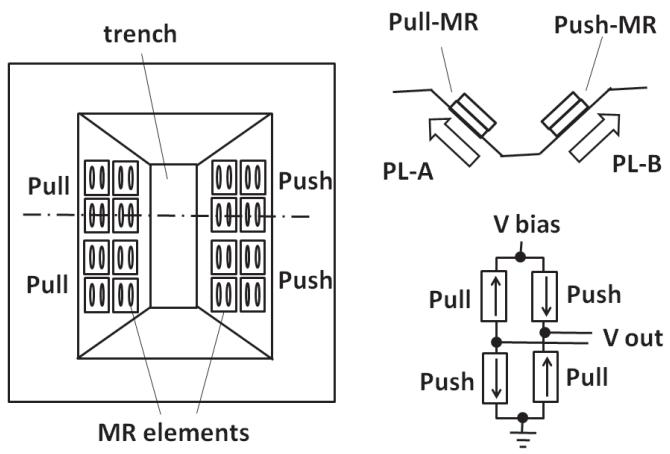

Fig. 5. An example a trench structure and MR elements used to make a push-pull Z-axis sensor bridge. PL-A, and PL-B represent the pinned layer set directions of the MR elements.

Another mechanical means used to make a ZAxis sensor from in-plane MR sensing elements is illustrated generically in Fig. 5. Many variations on this theme are possible including different wiring arrangements, different types of sensor elements, or the trenches may be replaced with bumps. In any case, in Fig 5, the MR sensors are grown on top of sloped surfaces which are cut into the substrate. The left-hand side of Fig. 5 shows a top down view of a representative design. The dashed cut-line across the trench defines the line along which the contour, illustrated in the top right of the figure is taken. The circuit diagram at the 
bottom right shows how the MR elements grown on the different slopes can be arranged into a full-bridge push-pull full bridge.

Note that the pinned layers of the MR elements on opposite sides of the trench illustrated in Fig. 5 must be aligned in different directions. This can be accomplished by magnetic annealing with a magnetic field in the Z-direction. [5] When interconnected as a bridge, the in-plane magnetic field cancels, and only the Z-axis field produces a push-pull response.

\section{Perpendicular Magnetic Anisotropy}
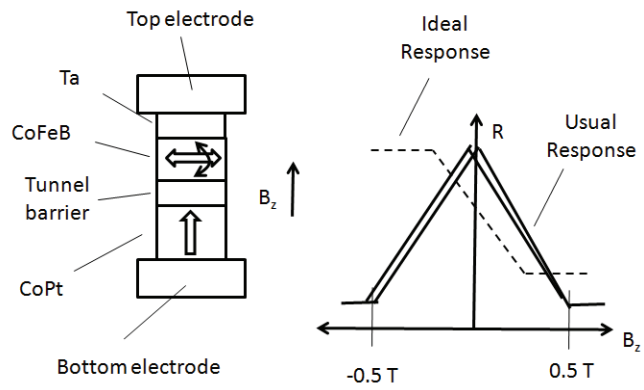

Fig. 6. An idealized PMA MTJ and ideal response curve.

An ideal solution for achieving Z-axis sensing with the MR effect would seem to be to use perpendicualr magnetic anisotropy (PMA) in order to permit the free magnetic layer to rotate into the out of plane direction and to set the pinned ferromagnetic layer into the perpendicular direction. Materials such as CoPt can have PMA and high coercivity, and thin layers of CoFeB coupled with an adjacent layer of Ta can have a PMA surface anisotropy. [6] A simple ideal illustration of the concept is shown in Fig. 6. Unfortunately, the saturation field is usually quite high for these materials, TMR is often reduced, and magnetic behavior is not ideal. [6,7]

\section{Flux Concentrators}

Instead of rotating the sensors into the Z-Axis direction or using large perpendicular anisotropy to compete against the shape anisotropy of the thin film, an alternative approach for sensing Z-Axis fields using TMR sensor elements has been developed that utilizes soft ferromagnetic flux concentrators in order to bend magnetic flux from the $Z$ direction into the in-plane direction that the MR sensors naturally detect. The concept for this approach is illustrated in Fig. 7, and it is described in detail in reference 4 . Here, a series of permalloy bars is placed over top an alternating array of push and pull TMR sensors. When a magnetic field is applied parallel to the Z-Axis, the flux is sucked into the parmalloy bars, and fringes outward along the corners of the permalloy bars in the figure. It can be observed that some of the flux in the vicinity of the MTJ elements fringes into the positive $\mathrm{X}$-direction, and some fringes into the negative $X$-direction. If the pinned layer of the MTJ elements is set along the X-Axis, then some MTJ elements will show increasing resistance, while others show decreasing resistance. Because of this effect, when the alternating rows of MTJ elements are interconnected as in the circuit diagram on the right hand side of Fig. 7, a push-pull bridge is formed.

A photograph of an exemplary layout is shown in Fig. 8. Here, several flux concentrator bars are used, and the rows of MTJ elements representing push and pull bridge arms are interleaved. This interleaving of the bridge arms reduces offset and improves temperature compensation of the sensor. This is because the bridge arms are not localized in one area so that process and thermal variation is more likely to affect each arm equally.

The magnetic performance of the resulting ZAxis sensor is shown in Fig 9. Here, the response of the Z-Axis sensor is measured as a function of magnetic field applied along the $X$, $Y$, and $Z$ directions, respectively. The small cross-axis response can be understood as follows. When a field is applied in the plane of the substrate, along the long direction of the flux concentrators, that is, along the Y-Axis, it is sucked into the concentrators, and thus the gaps between the concentrators where the MTJs reside are strongly shielded. There is thus little response. When a field is applied along the X-Axis direction, the push and pull MTJs show the same change in resistance, and provided the bridge is well balanced, the full bridge produces no output.

By adjusting the sensor geometry and MTJ materials, it has been found that the sensitivity of the device can be adjusted from less than 0.2 $\mathrm{mV} / \mathrm{V} / \mathrm{Oe}$ to over $3 \mathrm{mV} / \mathrm{V} / \mathrm{Oe}$, with saturation fields ranging from over 1000 Oe to less than 50 Oe. The design can therefore be tuned to operate over wide field range, similar to a Hall Effect sensor to a narrow field range, compatible with high precision compassing applications. 


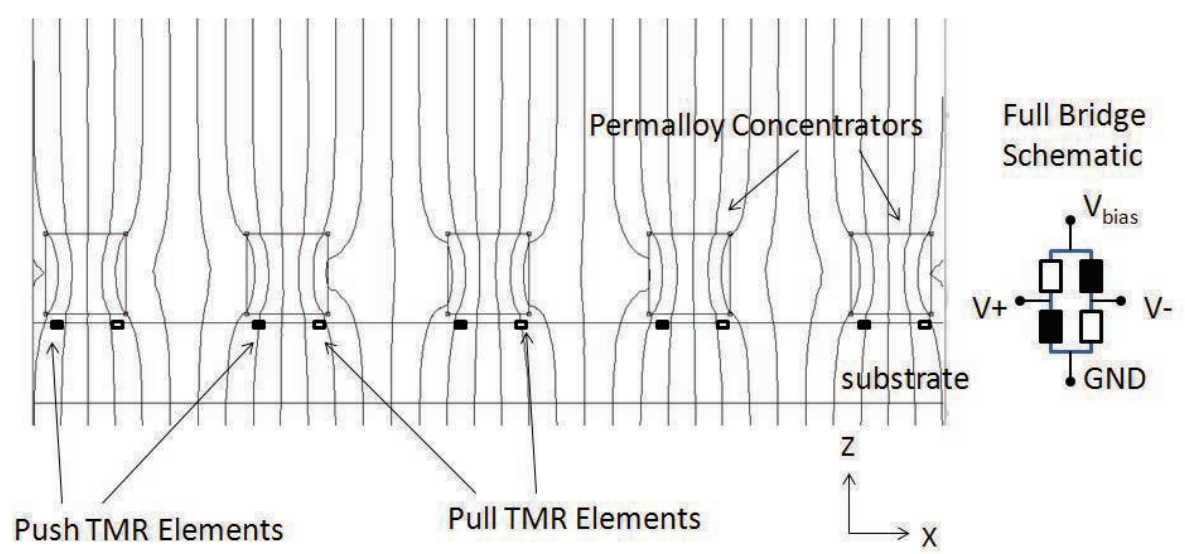

Fig. 7. A schematic cross-section drawing of a Z-Axis sensor chip showing the magnetic field distribution around the flux concentrator array when a field is applied parallel to the Z-Axis. The inset on the right shows the interconnection of the push (black) and pull (white) MTJ sensor elements to form a push-pull full-bridge magnetic sensor.

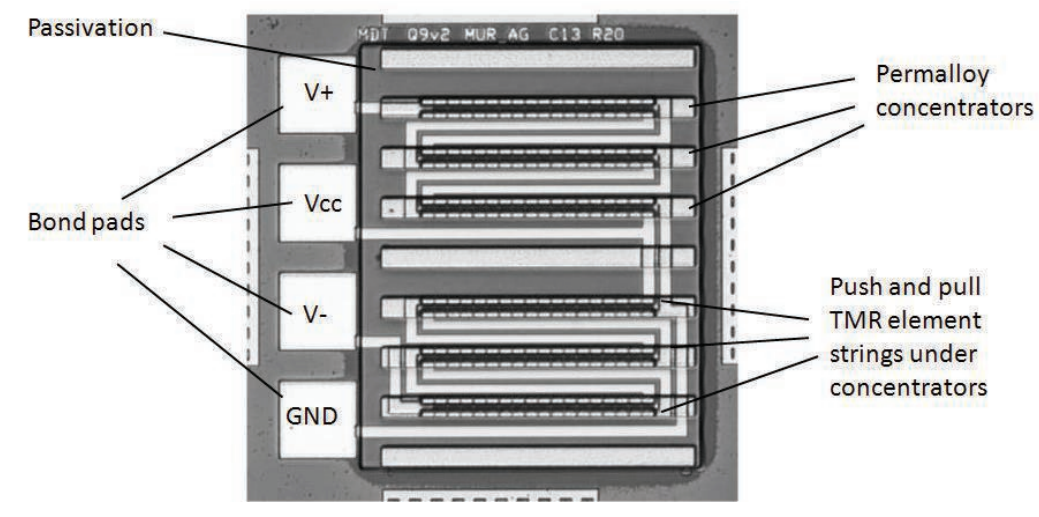

Fig. 8. A photograph of a TMR Z-axis sensor die. The die is $<0.5 \mathrm{~mm}$ on edge.

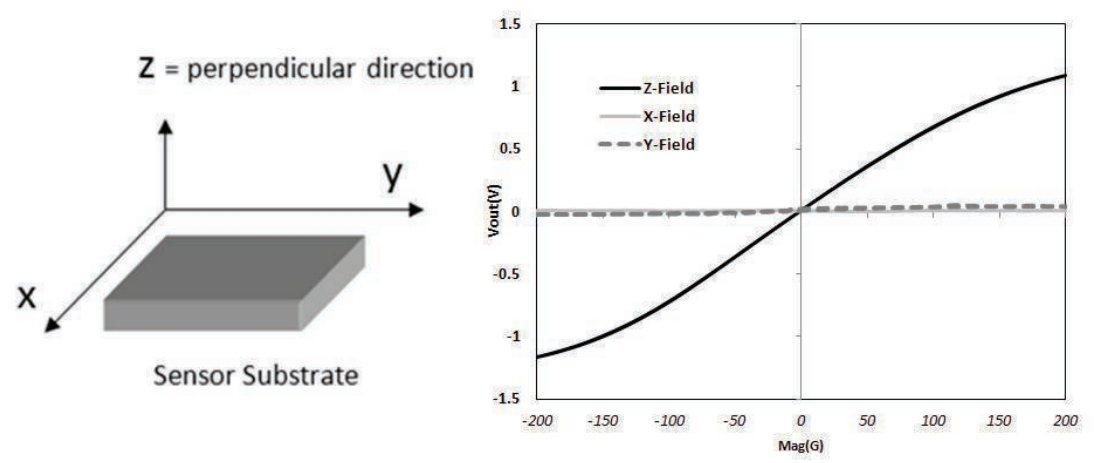

Fig. 9. The measurement geometry and representative voltage vs. applied magnetic field transfer curves measured on a test structure. The sensor is biased at $3 \mathrm{~V}$, and magnetic fields are swept along the $X, Y$, and $Z$ directions. Clearly the sensor is only sensitive to the Z-axis magnetic field and insensitive to in-plane magnetic field. 
In addition to having higher sensitivity than Hall Effect sensors, when the TMR Z-Axis sensors are optimized for a wide field range, they have some important advantages compared to Hall Effect sensors.

Because the resistance of MTJ elements is exponentially dependent on the thickness of the tunnel barrier, the resistance of the TMR Z-Axis sensor can be tuned over a wide range, without changing the magnetic performance of sensor. These sensors have been built with resistance ranging from $5 \mathrm{k} \Omega$ to over $1.5 \mathrm{M} \Omega$, without affecting magnetic performance. This makes it possible to produce a Z-Axis sensor that has much lower power consumption than a Hall Effect sensor, and low power can be achieved without duty-cycling the sensor, greatly improving read speed or reducing system design complexity.

Equally important, the temperature performance of TMR sensors is superior to Hall Effect sensors. Fig. 10 shows the typical temperature dependence of the output of a TMR Z-Axis sensor. The sensor is biased at 500 Oe applied along the Z-Axis, and the output is measured at temperature ranging from $-55 \mathrm{C}$ to $150 \mathrm{C}$. Over this temperature range, the sensitivity drifts less than $1.4 \% / C$. A good Hall Effect sensor, such as an Asahi-Kasei Microdevices HW322B for example, is rated at a typical value of $1.8 \% / \mathrm{C}$ over a range of $-40 \mathrm{C}$ to $110 \mathrm{C}$. The TMR Zaxis sensor shows smaller drift, and it can operate over a wider temperature range.

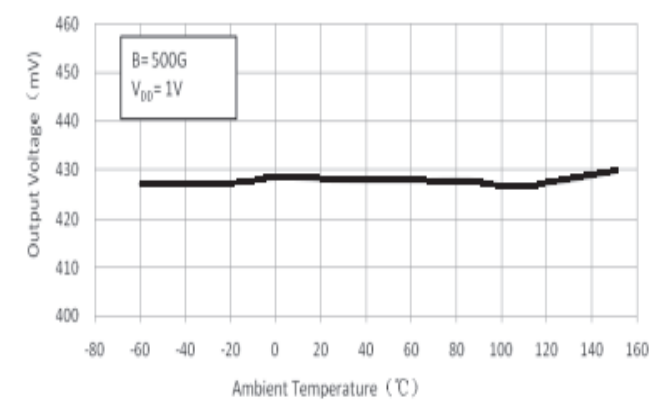

Fig. 10. Output voltage of a TMR Z-Axis sensor as a function of ambient temperature. The sensor is biased at $500 \mathrm{Oe}$ in this test.

\section{Conclusion}

A new method for producing a TMR Z-Axis magnetic field sensor has been presented. The TMR Z-Axis sensor is easier to fabricate than conventional methods involving mechanically rotating a MR sensor chip or depositing and patterning MR elements on a sloped surface etched into a substrate. The design provides flexibity to tune the sensitivity, measurement range, and resistance over a wide range. The resulting sensors can be made competitive with Hall Effect sensors in many applications while maintaining superior sensitivity and temperature performance. The design may also be tuned for high sensitivity and easily integrated on a single chip with in-plane MR sensors in order to produce a three-axis compass. The design is compatible with AMR, GMR and TMR effects, but because of the higher magnetoresistance of the TMR effect, it is best suited for TMR.

\section{References}

[1] H. Wan, R. Reiger, and M. Bohlinger, United States Patent Application US 2009/0072823 A1(2009)

[2] A. Peczalski, et. al., United States Patent US7126330 B2 (2006).

[3] J. G. Deak, Chinese Patent CN203480009 U (2013).

[4] F.Kou, A. Fuse, and J. Azumi, European Patent Application 09250711.0 (2009).

[5] S. Ikeda, et al., Nature Materials 9, 721-724 (2010); doi:10.1038/nmat2804

[6] J. E. Davies, United States Patent 8,773,821 (2014).

[7] S. van Dijken and J. M. D. Coey,, Appl. Phys. Lett. 87, 022504 (2005); doi:10.1063/1.1957111 